\title{
Should Seed Be an Alternative Source of Camptothecin From Nothapodytes nimmoniana and Chonemorpha fragrance?
}

\author{
Tasiu Isah
}

\section{Tasiu Isah}

Department of Botany, Hamdard University New Delhi 110 062, INDIA.

\section{Correspondence}

Tasiu Isah, Department of Botany, Hamdard University, New Delhi 110 062, INDIA.

Telephone: +919650487083

E-Mail: taasmore@yahoo.co.uk

DOI : 10.5530/pj.2016.6.11

Article Available online

http://www.phcogj.com/v8/i6

Copyright

(C) 2016 Phcog.Net. This is an open-

access article distributed under the terms of the Creative Commons Attribution 4.0 International license.

\begin{abstract}
The Southeast Asian forest is blessed with plant sources of anticancer molecules including camptothecin (CPT) but, the irrational harvest is among serious threat to its existence for use by the future generation. Among plant sources of CPT found in the region are Nothapodytes nimmoniana and Chonemorpha fragrance that are under heavy exploitation pressure for the alkaloid and other medicinal purposes. Under the natural conditions of forests, seeds produced by the plants show germination difficulties, and as a result lose viability few months after set. The unviable seeds can be used as an alternative source of CPT and other phytochemicals isolated from the species. In this study, evaluation of CPT yield of seeds and their parts was performed by high-performance thin layer chromatographic analysis to assess the feasible economic use of the seeds as an alternative source of the alkaloid. The results showed a manifold higher yield of CPT in seeds and their parts in $N$. nimmoniana over $C$. fragrance, suggesting seeds of the former that face more exploitation of its forest than the latter as a better alternative source of the alkaloid. The results also suggest that collection of the seed should be made before the onset of rain season. In this study, the economic implications of using seeds to isolate CPT are also discussed.
\end{abstract}

Key words: Conservation, Forest Management, Camptothecin, Seeds, Nothapodytes nimmoniana, Chonemorpha fragrance.

\section{INTRODUCTION}

Forestry is a vital source of natural anticancer molecules but, the irrational use in Southeast Asian countries is of serious concern on the availability to the future generations. The medicinal importance of a forest plant lies on the bioactive constituents it produces that have significant pharmacological activity and can be isolated with potentials for drug development. ${ }^{1}$ The breakthrough on cytotoxicity of extract from plants led to the discovery of natural compounds with anticancer properties ${ }^{2}$ and their development into drugs emanated from studies of extracts isolated from seeds. ${ }^{1,2}$ Biosynthesis and yield of the compounds vary with forest species and with many, seeds are an important source of the alkaloids. They offer a practical, cost-effective alternative to extraction from the natural population over chemical synthesis. Among high-value alkaloids isolated from native Southeast Asian forest plants is camptothecin (CPT) which is a monoterpene indole anticancer alkaloid from which many semisynthetic analog drugs used to treat varied human cancer types were developed.

Nothapodytes nimmoniana is an endangered medicinal forest tree and most convenient source of the alkaloid. ${ }^{3}$ The tree belongs to family Icacinaceae and is found in Sri Lanka, China (East and Mainland) Mainland
South East Asia, North Sumatra, Sundaic region in Taiwan, Isabela province, Luzon Philippines and India where the endemic distribution in the Western Ghats is under heavy exploitation for CPT. ${ }^{4}$ In the region, because one ton of the alkaloid can be isolated from 1000-1500 tons of N. nimmoniana wood chips, the natural population is overharvested for export of CPT. This led to declined forested area and tree individuals by $50-80 \%$ in the last decade. ${ }^{5}$ The loss in areas of patchy endemic distribution have remarkably declined to 1-2 individuals/hectare and the trend is ever on the increase. ${ }^{6}$

Chonemorpha fragrance is a seed-propagated evergreen vigorous growing endemic and endangered climbing shrub that produces stem of $30 \mathrm{~m}$ or more and can climb the top of the tallest tree in a forest. The plant produces many phytochemicals attributed the many biological activities exhibited by extracts isolated from different parts of the species. ${ }^{7}$ Campto-thecin was recently isolated from the climber with subcellular localization identified. ${ }^{8-9}$ Although the alkaloid is produced at low yield in Chonemorpha fragrance, it is among endangered natural sources that could face over harvesting pressure for CPT.

As CPT is isolated at higher yield from N. nimmoniana with greater quantity in stem and 
root of the tree than other natural sources, its natural population is under indiscriminate exploitation for the alkaloid. Annual demand for wood chips for domestic consumption and export reached 500700 metric tons. ${ }^{6}$ The tree produces many and desiccations sensitive seeds bearing small embryo with low germination capacity and unviable seeds can be found in large numbers on forest floor few months after seed set. ${ }^{10-11}$ Although $C$. fragrance produces CPT, albeit at alow yield, the species is propagated via seeds. This, coupled with slow growth in nature makes natural population subject to overexploitation for CPT with consequence on declined number of individuals and current endangered status in the Western Ghats, India.

Cultivation of CPT-producing plants as cash crops is receiving increasing consideration in Southeast Asia where many endemic species produce the alkaloid. ${ }^{12,13}$ The over-exploitation of their natural population for domestic use and export of CPT is ever on the increase. Threats due to recalcitrant seeds produced by the plants and slow natural growth led to increasing the call for conservation by implementing strategies against exploitation at the local level. ${ }^{14-15}$ Promoting the use of non-destructive parts as seeds to isolate CPT could be a promising alternative. The recalcitrant seeds produced by the plants may be considered an alternative source of CPT when they lose viability as many could be found on forest floor few months after seed set. Although in the earlier studies, CPT yield in seed mass of N. nimmoniana found in India was quantified ${ }^{16-21}$ and new camptothecinoids isolated in Taiwan..$^{22}$ The various parts of the seeds and variability in yield of the alkaloid across seed sources to (or "intending to") assessing workable economic use for CPT extraction were not analyzed. For C. fragrance, no study investigated the production or evaluation of CPT yield in the seeds and their parts. In this study, potentials and possibility of using seeds as an alternative source of CPT from $N$. nimmoniana and C. fragrance are evaluated and discussed.

\section{MATERIALS AND METHODS}

\section{Seeds collection}

Authenticated seeds of $C$. fragrance was obtained from Indian Institute of Horticultural Research Bengaluru, Karnataka India while N. nimmoniana seeds from three areas in the Western Ghats; One of the seed samples was provided by Sanjivini herbals Salem, Tamil Nadu India (S1). The other samples by Rishi herbals Bengaluru, Karnataka (S2) and Mr. N. Balakumar of Karthik Agro Traders Allagapaturam Kathoor Tamil Nadu (S3). Dried seeds of both of the species were soaked in sterile distilled water overnight to soften seed coat for the ease of seed embryo axes removal from the seeds. The excised seed embryo axes were used for alkaloid extraction after dried to constant mass or further experiment $\mathrm{s}^{23}$ while seed coat dried to constant mass. Fruit wall of the seeds was removed using forceps before seed soaking in water.

\section{Alkaloid Extraction and High-Performance Thin Layer Chromatographic analysis}

Camptothecin extraction was performed using microwave irradiation method of Fulzele and Satdive ${ }^{17}$ with slight modifications. In brief, various components of the seeds comprising dried seed coat, fruit wall, seed embryo axes and whole seed mass of $C$. fragrance and $N$. nimmoniana were dried to constant mass. One gram of the dried samples was ground to fine powder using mortar and pestle and transferred into a $200 \mathrm{ml}$ glass beaker, and $50 \mathrm{ml}$ of $90 \%$ HPLC grade methanol added to the contents. The beaker containing the suspension were subjected to microwave oven extraction at $100 \mathrm{w}$ for 3 mins. Obtained suspensions were not allowed to super-boil in the micro wave by the regular stoppage of the irradiation and to cool the beaker (with contents) in water for minutes. Contents were again subjected to microwave irradiation and cooling cycles to complete three rounds. Suspensions obtained from the extraction were allowed to settle and upper aqueous phase transferred into 2 ml-capacity centrifuge tube, and then centrifuged at $14,000 \mathrm{xg}$ for $10 \mathrm{mins}$. Obtained supernatants were filtered using a Millipore filter $(0.45 \mu \mathrm{M})$ into a fresh centrifuge tube. High-Performance Thin Layer Chromatographic (HPTLC) analysis was performed using modified method of Kulkarni et al. ${ }^{8}$ Briefly, $20 \mu \mathrm{l}$ of the extracts were loaded to an HPTLC plates (Merck silica gels) using automated sample loading system (Linomat 5 Camag). The applied samples were allowed to dry for minutes and later developed in duplicates in a glass chamber saturated with ethylacetate:toluene (7:3) mobile phase solvent system. Developed chromatographic plates were dried and later scanned with Camag 3 densitometric scanner. The peaks, peak area and $\mathrm{Rf}$ of the spots recorded. Samples of pure CPT standard was procured from Sigma Aldrich and working stock solution prepared by dissolving $800 \mu \mathrm{g}$ of CPT powder in $1 \mathrm{ml}$ chloroform:methanol solvent mixture (4:1). Different working concentrated dilutions were prepared from a standard stock solution. They were then spotted on a TLC plates (silica Merck), air-dried for minutes and allowed to develop in a saturated chromatographic glass chamber. The procedure was used for the CPT reference standard dilutions in a similar way to plant tissue extract. The experiment was carried out in duplicates, the fluorescence and Rf of CPT recorded at $366 \mathrm{~nm}$. Camptothecin yield of the various tissues was evaluated from a standard curve drawn using different concentrations of the pure camptothecin reference standard.

\section{RESULTS AND DISCUSSIONS}

In the Southeast Asia, conservation of forest genetic resources, particularly those that produce bioactive molecules with anticancer properties is receiving increased recognition due to recent awareness on the threat to their availability for the future generation. ${ }^{24}$ Besides a primary role in sexual propagation; plant seeds can be a valuable source of bioactive compounds, including anticancer alkaloid CPT. However, seeds are among neglected sources of the alkaloid due to their status in plants propagation and more preference given to the root, shoots/wood and leaves for alkaloid isolation. This has lead to a remarkable loss in plant genetic resources in forested areas due to exploitation for the molecule. In recent years, alternative collection measures are increasingly sought. Although establishing protected areas is a conservation strategy used to protect the genetic resources in some of the countries, it does not reduce irrational harvest of forest for CPT. ${ }^{25}$

Hard seed coat and dormant embryos, coupled with difficult-to-get favorable environmental conditions of moisture, oxygen, and soil hinders seed germination with many of the forest plants. As a result, they lose viability within a short period after set, in particular, those that produce recalcitrant seeds. Thus, the seeds that represent a 'waste of propagules' become available for other economic use, including extraction of the important phyto-compounds as CPT. ${ }^{26,27}$ During the reproductive season, $N$. nimmoniana produces many seeds but, due to natural recal citrance, majority lose viability before the onset of germination season when enough moisture become available to support germination, seedling cohorts establishment and forest generation. ${ }^{28}$ The unviable seeds found in large numbers on the forest floor are of no use for propagating the tree, and overtime, they become attacked by fungi (personal communication with locals). C. fragrance produces a large number of seeds but, due to hard seed coat (Figure 1a), germination under the natural condition is poor. As a result, the majority do not germinate in a forest and over time, they lose viability. Promotion of seeds collection for alkaloid extraction and export is a significant step towards mitigating irrational harvest of the two species for CPT. For instance, in N. nimmoniana seedlings need 6-8 years to reach maturity and reproductive stage. ${ }^{6}$ A collection of the entire mature tree may not yield equivalence of CPT produced by seeds over the eight year period as the seeds are produced in large number by 


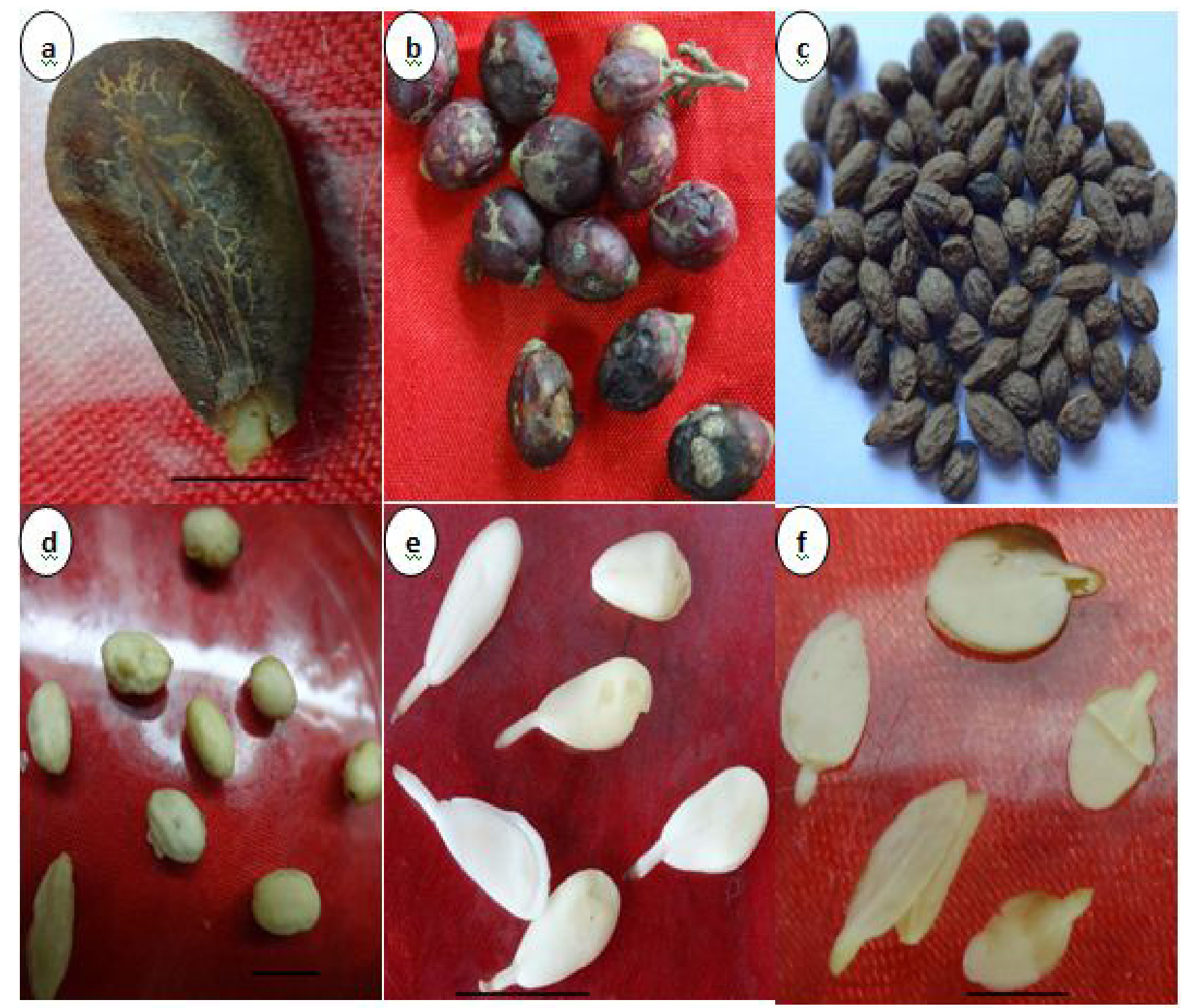

Figure 1: a, C. fragrance seed mass; b, N. nimmoniana fruits; c, N. nimmoniana dried seed; d, decorticated N. nimmoniana seeds (Scale bar $2 \mathrm{~mm}$ ); e, excised seed embryonal axes of C. fragrance (Scale bar $2 \mathrm{~mm}$ ); f, excised seed embryonal axes of N. nimmoniana (Scale bar 1.5mm).

the tree in a season. Assessing the yield of the alkaloid in seeds and their parts could give an insight on potential economic use to isolate CPT.

Besides a primary role in plant reproduction/propagation, seeds could be an alternative source of CPT to other plant parts as leaves, shoots/ wood, and roots, based on the pattern of accumulation of the alkaloid. However, the implication of differential alkaloid yield from the seeds and other plant parts is unknown in many forest trees. ${ }^{29}$ To evaluate the potential of the seeds as an alternative source for extraction of CPT and as a substitute to the harvest of natural stands, alkaloid was extracted from the ground seeds of N. nimmoniana and C. fragrance, their various parts and obtained tissue extracts analyzed by HPTLCanalysis (Figure 1a-f, 2b-c). The analysis showed manifold CPT content in seeds and their part in N. nimmoniana over C. fragrance (Figure 2a), suggesting that the former has more potential as an alternative source of the alkaloid than the latter. The highest yield of the alkaloid was found in seed mass of N. nimmoniana with greatest CPT yield of $0.16 \% \mathrm{CPT} / \mathrm{g}$ seed DW over $0.0097 \%$ in C. fragrance. The yield varied with seed source and highest found in seeds obtained from Sanjivini herbals while least in those from Karthik Agrotraders Tamil Nadu. This showed the genotype-dependent effect on CPT content in N. nimmoniana seeds. Earlier studies on populations of the species in the Western Ghats region, India showed variability in CPT yield in wood and shoots of individuals populations in a forest. ${ }^{30-31}$ The yield of the alkaloid in the seeds used in this study is less than reported for wood in the reports. Although the yield of the alkaloid from seeds of a plant depends on plant habit, climatic condition, genetic make up and proportion of species producing the alkaloid, and may not depend on latitude. ${ }^{32-33}$ In this study, CPT yield of N. nimmoniana seeds varied across sources (Figure 2a) which could be due to the influence of climatic condition as biosynthesis of plant secondary metabolites that includes CPT are influenced by environmental factors. In C. acuminata that produces higher CPT in seeds over seedlings, collected seeds in different geographical regions showed variation in CPT yield, and the variability reflected seed length, stem diameter and height. ${ }^{34}$ From the results of this study, CPT yield in N. nimmoniana and C. fragrance was 


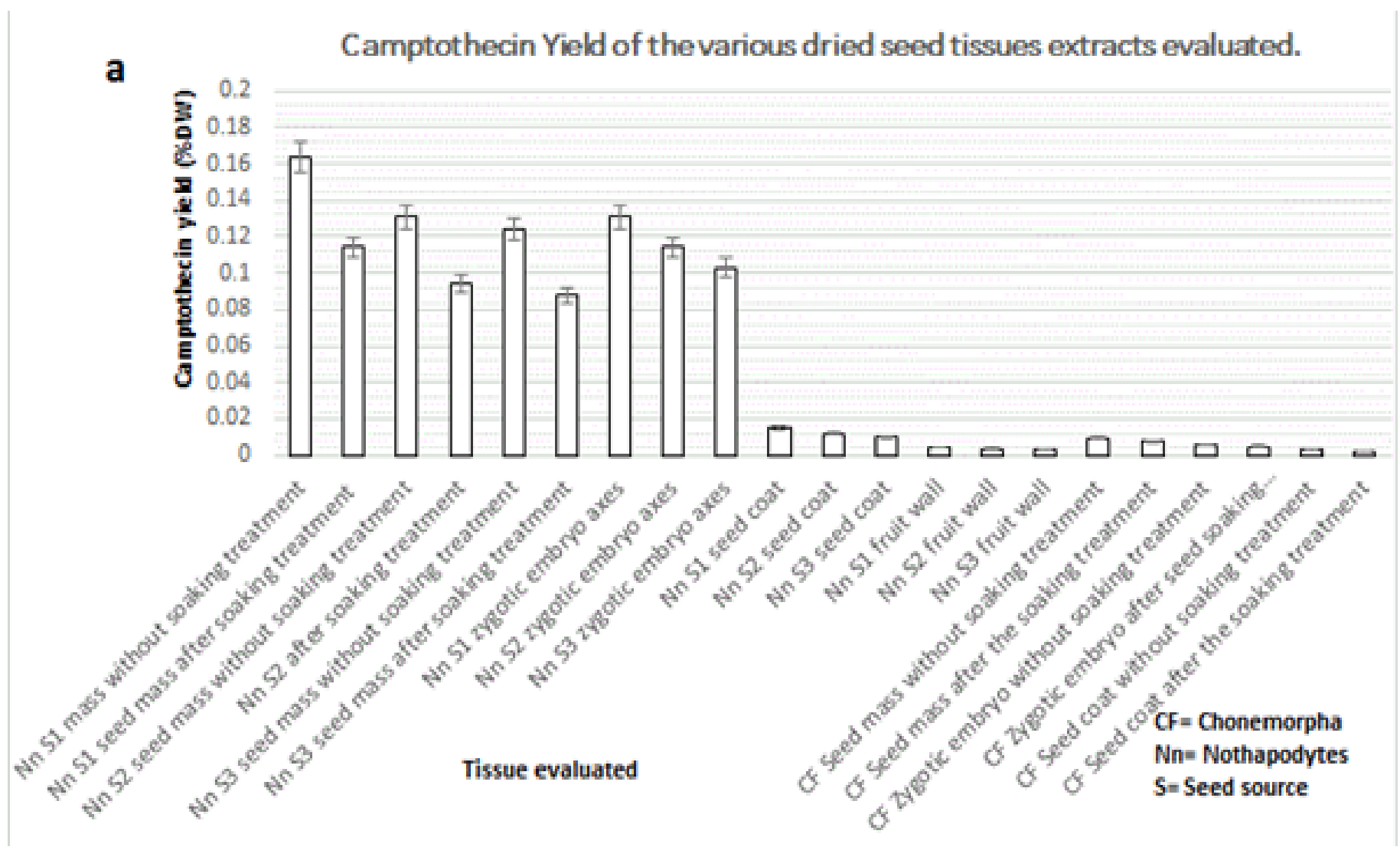

b

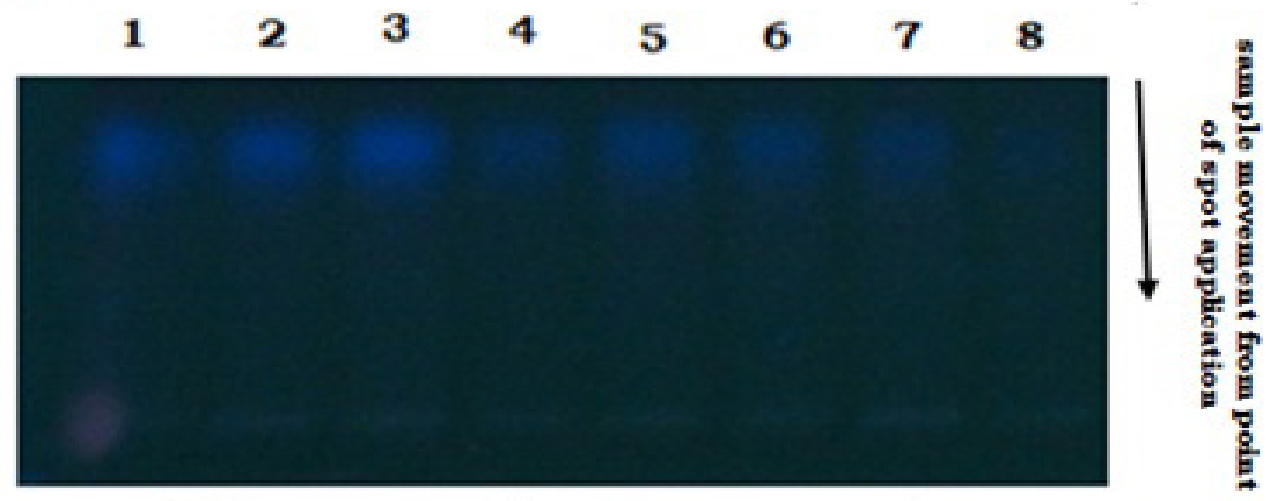

UV fluorescence at $366 \mathrm{~nm}$ showing presence of

CPT at variable concentration in the seed tissues analzed.

Figure 2: (a) Camptothecin yield of the tissues evaluated; (b) representative UV fluorescence of CPT at 366nm in some of the seed tissue samples analyzed..

affected by pre-soaking treatment in water overnight as shown by the lower CPT content of the seeds subjected to overnight pre-soaking (Figure 2a). This can be explained by the leaching of the alkaloid in water as CPT is water-soluble; ongoing study with cell suspension culture of the species showed that high amount of the alkaloid is leached into culture media (unpublished). Also, from the results, it can be suggested that collecting seeds of the two species (particularly, those that lost viability) from forest should be done before the onset of rain season for greater CPT yield.

For evaluating CPT yield in various seed parts of the two species, fresh fruit wall, seed coat, and seed embryo axes were dried separately, the alkaloid was extracted and HPTLC analysis performed (Figure $2 \mathrm{~b}-\mathrm{c}$ ). In a similar way to seed mass, CPT yield was manifold higher in various seed parts of N. nimmoniana over C. fragrance. The yield varied with seed source in N. nimmoniana with the highest found in seeds obtained from Sanjivini herbals Tamil Nadu when compared to the other sources (Figure 2a). A comparison of various parts showed seed embryo contained higher CPT over seed coat and fresh fruit wall. However, combined CPT yield of the seed parts was near to seed mass (Figure 2a). The little differences observed may have been caused by extraction technique conditions and pre-soaking treatment of the seeds in water. Compared to the seed embryo of $C$. fragrance where greatest of $0.0053 \%$ CPT yield was 
found, CPT yield of seed embryo axes of N. nimmoniana was relatively higher with $0.131 \%$ CPT. The yield varied with seed embryo axes seed source as well (Figure 2a). Camptothecin content of seed coat was manifold higher in N. nimmoniana over C. fragrance and varied with seed coat seed sources (Figure 2a). The yield was lower in the seed coat of C. fragrance seeds subjected to pre-soaking treatment in water overnight. This further established the effect of seeds soaking in water to reduced CPT yield in the two species. There was very low level accumulated CPT in fresh fruit wall of $N$. nimmoniana seeds and was variable with seed sources as well (Figure 2a).

From results of this study and the reported literature on CPT yield of seeds, their parts, and in various plant parts of the two species ${ }^{15}$ it can be inferred that seeds represent a practical alternative source of CPT, especially the ones that have lost viability.Thus, a strategy for conservation of the two species that involves using the unviable seeds (as they become a waste in the forest after germination season passed) for extraction of $\mathrm{CPT}$, and as raw material for export, is an important conservation strategy. The introduction of saplings into new sites to set up forested areas and legislations regulating overharvest of the endangered natural population is an important step. Driven by demand for CPT in the international trade, commercial farmers in some states in India have developed plantations of $N$. nimmoniana to (or "intending to")cultivating the tree parts for export while in other Southeast Asian countries, no such efforts are made. For C. fragrance effort towards forestation or establishment of commercial cultivation is none existent so far, possibly due to the low $\mathrm{CPT}$ yield but, the population is continuously exploited for other medicinal uses.

\section{ACKNOWLEDGEMENT}

The author acknowledge Department of Biotechnology Government of India and The World Academy of Science for The Advancement of Science in Developing Countries for financial support.

\section{CONFLICT OF INTEREST}

The author do not have conflict of interest on the manuscript contents.

\section{REFERENCES}

1. Cragg GM, Newman DJ. Nature: a vital source of leads for anticancer drug development. Phytochem Rev. 2009;8(2):313-31. http://dx.doi.org/10.1007/ s11101-009-9123-y.

2. Wall ME, Wani MC, Cook CE, Palmer KH, McPhail AT, Sim GA. Plant Antitumor Agents I. The Isolation and Structure of Camptothecin, a Novel Alkaloidal Leukemia and Tumor Inhibitor from Camptotheca acuminata. J Am Chem Soc. 1966;88(16):3888-90. http://dx.doi.org/10.1021/ja00968a057.

3. Govindachari TR, Viswanathan N. Alkaloids of Mappia foetida. Phytochem. 1972;11(12):3529-3531. http://dx.doi.org/10.1016/S0031-9422(00)89852-0.

4. Kårehed J. Trees of Tropical Asia Old Icacinaceae. Am J Bot. 2001;88:2259-74. PMid:21669659

5. Kumar R, Ved DK. Illustrated field guide to 100 red-listed medicinal plants of conservation concern in Southern India. Foundation for Revitalization of Local Health Traditions Bangalore. 2000:261-3.PMid:10741592.

6. Patwardhan A. Domestication of Nothapodytes nimmoniana (Grah.) Mabb., an endangered medicinal tree from the Western Ghats of India. The Rufford Small Grants Foundation, UK, 2006:7-15.

7. Amrish C, Rekha R. Chonemorpha fragrance, An endangered medicinal plant: a review. Intl J Pharm Erud. 2011;1(3):10-6.

8. Kulkarni AV, Patwardhan AA, Lele U, Malpathak NP. Production of camptothecin in cultures of Chonemorpha grandiflora. Pharmacog Res. 2010;2(5):296-9. http://dx.doi.org/10.4103/0974-8490.72327.

9. Kedari P, Malpathak N. Sub-cellular localization and quantification of camp to the cin in different plant parts of Chonemorpha fragrance. Advances Zool Bot. 2013;1 (2):34-8

10. Sharma SN, Puri SC, Srivastava TN, Handa G, Kaul BL. Enhancement of seed germination in Nothapodytes foetida. J Med Aromatic Plant Sci. 2000;22(2/3):206-10

11. Sharma MV, Shaanker RU, Leather SR, Vasudeva R, Shivanna KR. Floral resources, pollinators, and fruiting in a threatened tropical deciduous tree. J Plant Ecol.
2011;4(4):259-267. http://dx.doi.org/10.1093/jpe/rtq029

12. Wu TS, Leu YL, Hsu HC, Ou LF, Chen CC, Chen CF, et al.Constituents and cytotoxic principles of Nothapodytes foetida. Phytochem. 1995;39(2):383-5. http:// dx.doi.org/10.1016/0031-9422(94)00901-5.

13. 13. Ramesha BT, Suma HK, SenthilKumar U, Priti V, Ravikanth G, Vasudeva $R$, et al. New Plant Sources of the anticancer alkaloid, camptothecin from the Icacinaceae taxa India. Phytomed. 2013;20(6):521-7. http://dx.doi.org/10.1016/j. phymed.2012.12.003.

14. 14. Isah T, Mujib A. In vitro propagation and camptothecin production in Nothapodytes nimmoniana. Plant Cell Tiss Organ Cult. 2015;12(1):1-10. http://dx.doi. org/10.1007/s11240-014-0683-1.

15. 15. Isah T, Mujib A. Camptothecin from Nothapodytes nimmoniana: a review on biotechnology applications. Acta Physiol Plant. 2015;37(6):1-14. http://dx.doi. org/10.1007/s11738-015-1854-3

16. Puri SC, Handa G, Gupta RK, Srivastavam TN, Somal P, Sharma SN. Quantitation of camptothecin in Nothapodytes foetida. J Indian Chem Soc. 1999;76(7):370-1.

17. Fulzele DP, Satdive RK. Comparison of techniques for extraction of the anticancer drug camptothecin from Nothapodytes foetida. J Chrom Anal. 2005;1063(1-2): 9-http://dx.doi.org/10.1016/j.chroma.2004.11.020.

18. Singh I, Kumaravadivel N, Gnanam R, Vellaikumar S. RP-HPLC analysis for camptothecin content in Nothapodytes nimmoniana, an endangered medicinal plant. J Med Plants Res. 2010;4(3)255-9

19. Namdeo AG, Sharma A. HPLC analysis of camptothecin content in various parts of Nothapodytes foetida collected on different periods. Asian Pac J Tropical Biomed. 2012;2(5):389-93. http://dx.doi.org/10.1016/S2221-1691(12)60062-8.

20. Karwasara VS, Nahata A, Dixit VK. A simple, rapid and sensitive spectrofluorimetric method for the determination of camptothecin. Orient Pharm Exp Med. 2012;12(2):151-6. http://dx.doi.org/10.1007/s13596-011-0053-z.

21. Lokesh P, Balasubramanya S, Arunadha M. Cost effective quantification of camptothecin and a comparative study of its content in Nothapodytes foetida and Ophiorrhiza mungos sourced from selected geographical locations. Orient Pharm Exp Med. 2014;14(2):87-92

22. Wu SF, Hsieh PW, Wu CC, Lee CL, Chen SL, Lu CY, et al. Camptothecinoids from the seeds of Taiwanese Nothapodytes foetida. Molecules. 2008;13(6):1361-71. http://dx.doi.org/10.3390/molecules13061361.

23. Isah T, Mujib A. Enhanced in vitro seedling recovery in Nothapodytes nimmoniana (J. Graham) Mabberly. Br Biotechnol J 2015c;6(1):35 http://dx.doi. org/10.9734/BBJ/2015/15368.

24. Dubey N, Kumar R, Tripathi P. Global promotion of herbal medicine: India's opportunity. Curr Sci. 2004;86(1):37-41.

25. Shivaprakash KN, Ramesha BT, Uma Shaanker R, Dayanandan S, Ravikanth G. Genetic Structure, Diversity and Long Term Viability of a Medicinal Plant, Nothapodytes nimmoniana Graham. (Icacinaceae), in Protected and Non-Protected Areas in the Western Ghats Biodiversity Hotspot. PLoS ONE. 2014;9(12):e112769. http://dx.doi.org/10.1371/journal.pone.0112769

26. Urgenc S, Cepel N. Species Selection for afforestation: Practical Fundamentals of Sowing and Planting. TEMA Foundation. Publication no.33, Istanbul, 2001.

27. Jaiswal $P$, Chaudhary S.Germination behavior of some trees and grasses of arid lands. Bull Nat Inst Ecol. 2005;15:201-5.

28. Envis. Plant of the month-Nothapodytes nimmoniana. Center for the conservation of medicinal plants. 2014;http://frlhtenvis.nic.in/KidsCentre/Plant_1755. aspx.

29. Zhang J, Yu Y, Liu D, Liu Z. Extraction and composition of three naturally occurring anti-cancer alkaloids in Camptotheca acuminata seed and leaf extracts. Phytomed. 2007;14(1):50-56. http://dx.doi.org/10.1016/j.phymed. 2006.11.004.

30. Padmanabha BV, Chandrashekar M, Ramesha BT, Hombe Gowda HC, Gunaga RP, Suhas $\mathrm{S}$, et al. Patterns of accumulation of camptothecin, an anticancer alkaloid in Nothapodytes nimmoniana Graham., in the Western Ghats, India: implications for identifying high-yielding sources of the alkaloid. Current Sci. 2006;90(1):95-100.

31. Ramesha BT, Amna T, Ravikanth G, Gunaga RP, Vasudeva R, Ganeshaiah KN, et al. Prospecting for camptothecines from Nothapodytes nimmoniana in the Western Ghats, South India: identification of high-yielding sources of camptothecin and new families of camptothecines. J Chrom Sci. 2008;46(4):362-368. http://dx.doi.org/10.1093/chromsci/46.4.362.

32. Jones Q, Earle FR. Chemical analyses of seeds. II. Oil and protein content of 759 species. Econ Bot 1966;20:127-155. http://dx.doi.org/10.1007/BF02904010.

33. Earle FR, Jones Q. Analysis of seed samples from 113 plant families. Econ Bot 1962;16:221-250. DOI 10.1007/BF02860181.http://dx.doi.org/10.1007/ BF02860181.

34. Liu Z, Adams JC. Seed source variation in camptothecin concentrations of nursery-grown Camptotheca acuminata seedlings. New For. 1998;16(2):167-175. http://dx.doi.org/10.1023/A:1006556231438. 
Cite this article : Isah T. Should Seed Be an Alternative Source of Camptothecin From Nothapodytes nimmoniana and Chonemorpha fragrance? Pharmacognosy Journal. 2016;8(6):587-592. 